\title{
LOS HERMANOS DE NIÑOS CON DISCAPACIDAD INTELECTUAL: DIFICULTADES Y FORTALEZAS ${ }^{1}$
}

\author{
ELISA GARCÍA ZAMORANO \\ JUAN PEDRO NÚÑEZ PARTIDO ${ }^{2}$
}

Fecha de recepción: junio 2018

Fecha de aceptación y versión definitiva: julio 2018

RESUMEN: El objetivo de este estudio es comprobar si existen diferencias en el ajuste psicológico de los hermanos de niños con sindrome de Down, autismo y grupo control y analizar si variables como el nivel socioeconómico, sexo, grado de problemas de conducta del hermano con discapacidad, orden en la jerarquía fraterna y la percepción de la calidad de su relación, influye en las fortalezas y dificultades de los hermanos sin discapacidad. Para ello se utilizaron los cuestionarios Strengths and Difficulties Questionnaire (SDQ) y la escala de problemas de conducta del Inventario para la Planificación de Servicios y Programación individual (ICAP).

PaLABRAS CLAVE: discapacidad intelectual; síndrome de Down; autismo; hermanos; ajuste psicológico; fortalezas y dificultades.

\section{Siblings of children with intellectual disability: strengths and difficulties}

ABSTRACT: The aim of this paper is to identify if there are differences in the psychological adjustment of siblings of children with Down Syndrome, Autism and a control group. In addition, it will analyse whether variables such as socioeconomic status, sex, degree of behavioural problems of the sibling with disability, order in the fraternal hierarchy and the perception of the quality of their relationship, affects the strengths and difficulties of siblings without disabilities. For this purpose, two questionnaires were used, the Strengths and Difficulties Questionnaire $(S D Q)$ and the behavioural problems scale of the Inventory for the Planning of Services and Individual Programming (ICAP).

KEY WORDS: intellectual disability; Down syndrome; autism; siblings; psychological adjustment; strengths and difficulties.

1 Texto derivado de Trabajo de Fin de Máster.

2 Estudiante titulada del Máster en Psicología General Sanitaria por la Universidad Pontificia de Comillas. Correo electrónico: elisagarciazamorano@gmail.com. Profesor del Departamento de Psicología de la Universidad Pontificia de Comillas. Correo electrónico: jnunez@comillas.edu. 


\section{INTRODUCCIÓN}

\subsection{LA DISCAPACIDAD INTELECTUAL EN LA ACTUALIDAD}

Atendiendo a los manuales, en el Diagnostic and Statistical Manual of Mental Disorders (DSM-5) la discapacidad intelectual se define como un trastorno que comienza durante el periodo de desarrollo e incluye limitaciones del funcionamiento intelectual y del comportamiento adaptativo en los dominios conceptual, social y práctico (American Psychiatric Association, 2013). La OMS estimó en 2010 en el Informe mundial sobre la discapacidad que alrededor del $15 \%$ de la población mundial sufría algún tipo de discapacidad (Organización Mundial de la Salud, 2011). En este informe no se ha concretado el índice de población con discapacidad intelectual, y en el intento de obtener una cifra aproximada en España, se ha calculado que aproximadamente un uno por mil de la población española presenta algún tipo de discapacidad intelectual (Plena Inclusión, 2017).

En el ámbito de las personas con discapacidad se ha producido un gran avance, tanto en el contexto legislativo y social como en el de los servicios, que se han visto incrementados (Dyke et al., 2009; Rumeu, 2009). La sociedad actual se encamina hacia una postura orientada a la inclusión, cambiando por ejemplo el término retraso mental por el de discapacidad intelectual e intentando delimitar las definiciones de discapacidad intelectual, evitando las connotaciones negativas que hace años abarcaban. Se está avanzando hacia una visión más sistémica que fomenta la calidad de vida familiar, incluyendo en este proceso no solo a los familiares, sino también a los profesionales de la salud y a los educadores (Watson, 2017). Pero, a pesar de los avances logrados, queda un largo camino por recorrer, pues hoy en día, en la sociedad se sigue estigmatizando a esta población, y los familiares de éstos tienen que hacer frente a la intolerancia social diaria que existe ante la discapacidad (Lizasoáin y Onieva, 2010).

\subsection{LAS RELACIONES FRATERNAS}

La discapacidad intelectual como cualquier otro trastorno o enfermedad no solo afecta al sujeto que la padece, sino también a su entorno: familiar, social, comunitario, etc. Por lo tanto, es imprescindible tener en cuenta a las personas de los subsistemas en los que el sujeto con discapacidad participa para la intervención. El sistema más cercano donde el niño se relaciona es el sistema familiar, y dentro de éste al igual que todas las familias con varios 
hijos, es de gran relevancia el subsistema fraternal, es decir, las relaciones que se establecen entre los hermanos.

En el ambiente fraternal como en todas las relaciones, se pueden encontrar distintos tipos de afinidades entre los hermanos. En la mayoría de los casos, los hermanos suponen un apoyo y una relación diferente a la que se establece entre padres e hijos y distinta a su vez de la que se establece normalmente con el grupo de pares (esto puede variar en función del caso). A pesar de que los padres no forman parte directa del sistema fraternal, éstos tienen una gran influencia en el desarrollo de las relaciones entre hermanos, ya que suelen ser la fuente más importante de apoyo en las relaciones emocionales de sus hijos, enseñándoles así a que éstos se regulen emocionalmente y aprendan a socializarse entre ellos (Harris, 2001).

En 1982, Stephen Bank y Michael Kahn se refirieron al vínculo fraterno a través de la capacidad de acceso. Esta relación comienza en la primera infancia, produciéndose una mayor capacidad de acceso cuando los hermanos son del mismo sexo o de edades similares, aunque a su vez cuanto mayor acceso existe entre hermanos, más posible es que la relación sea más íntima y, por lo tanto, la probabilidad de que existan conflictos también se incrementa (Harris, 2001). Por ejemplo, es más común que surjan sentimientos de celos y rivalidad cuando los hermanos son similares en edad y en sexo que cuando no lo son, ya que al concebirse como más semejantes aparecen las comparaciones.

\subsection{EXPERIENCIA FRATERNA CON UN HERMANO CON DISCAPACIDAD INTELECTUAL}

Sin embargo, aunque el estudio de las relaciones fraternas puede ser similar en todos los casos, cuando el hermano presenta discapacidad intelectual, esta experiencia puede ser algo diferente a las relaciones fraternas normativas. En este aspecto, se han realizado numerosos estudios sobre la adaptación y el duelo en los padres de los niños con discapacidad, dejando en la mayoría de los casos a los hermanos en un segundo plano y apelando a ellos como «los grandes olvidados» (Hallberg, 2013; Núñez y Rodríguez, 2004; Watson, 2017). Es significativo resaltar que en todos los estudios se menciona la necesidad de incrementar las investigaciones en este campo para facilitar y mejorar la calidad de vida de todos aquellos afectados. También destacan la importancia de conocer cómo afecta a los hermanos de niños con algún tipo de trastorno el que su hermano lo padezca, con todas las consecuencias negativas o positivas que ello conlleva: sentimiento de soledad, impotencia, falta de atención, habilidades para la resolución de 
problemas, resiliencia... para así proporcionar al ámbito clínico información sobre el clima familiar existente y dotarle de la oportunidad de intervenir en aquellos aspectos que los miembros de la familia (en este caso el hermano del afectado) necesitan (Hastings, 2007; Rumeu, 2009).

Un niño con una enfermedad o trastorno puede provocar en los padres sentimientos negativos como agotamiento o frustración, los cuáles pueden verse desaprobados socialmente en la relación padres-hijo. Por tanto, teniendo en cuenta la dificultad que esto conlleva, cabe esperar que en el caso de los hermanos suponga un reto aún mayor, por ser estos más pequeños y tener menos habilidades de regulación emocional. De hecho, en algunos casos, los hermanos de niños con enfermedad pueden ocultar sus sentimientos por miedo de no ser aceptados por sus padres si manifiestan estas emociones negativas hacia su hermano. Por ejemplo, un niño puede sentirse egoísta al decirle a sus padres que quiere que pasen más tiempo con él cuando éstos están desbordados por cuidar del hermano afectado. Por ello, es importante conocer las vivencias de estos hermanos que muchas veces quedan reprimidos y aprobar la expresión de estos sentimientos, validándolos y enseñándoles a manejarlos (Núñez y Rodríguez, 2004). Muchos autores no solo muestran la necesidad de ayudar a validar los sentimientos a estos "grandes olvidados», sino también la importancia de compartir estas emociones en un ambiente de iguales y que se puedan sentir identificados y entendidos por personas como ellos para que el ajuste psicológico sea mayor (Aparicio y Mínguez, 2015; Iriarte e Ibarrola-García, 2016; Lizasoáin y Onieva, 2010; Rumeu, 2009).

La experiencia de tener un hermano con discapacidad varía dependiendo de la persona, observándose diversidad de experiencias positivas y negativas relatadas por los hermanos. Incluso, en muchos casos, aun valorando la vivencia de la discapacidad como algo positivo, pueden experimentar a su vez emociones negativas de vez en cuando, pudiendo crear ambivalencias de sentimientos (Iriarte e Ibarrola-García, 2016). Desde una vivencia más positiva, los estudios muestran que los hermanos destacan que tener un hermano con esta problemática les ha aportado mayor sentido de justicia, conciencia de las diferencias, afrontamiento para superar situaciones de estrés futuras, comprensión, autoestima por la adquisición de responsabilidades que no son propias para su edad, aceptación y en líneas generales un mayor grado de madurez que los hermanos de niños sin este diagnóstico (Aparicio y Mínguez, 2015; Hallberg, 2013; Iriarte e Ibarrola-García, 2016; Moyson y Roeyers, 2012; Watson, 2017). Sin embargo, existen también estudios que muestran emociones negativas asociadas a este duelo como es el aislamiento social percibido en los hermanos de estos niños, vergüenza por lo que 
puedan pensar sus amigos o la sociedad, envidia por las diferencias de trato que recibe su hermano/a con discapacidad, ansiedad y preocupación ante el futuro de éste, impotencia de no poder hacer nada para curar a su hermano o evitar el sufrimiento familiar o las tensiones familiares, rabia por ser ellos «la familia elegida», etc. (Aparicio y Mínguez, 2015; Hallberg, 2013; Iriarte e Ibarrola-García, 2016; Lizasoáin y Onieva, 2010; Moyson y Roeyers, 2012; Watson, 2017).

A pesar de esta variabilidad en las vivencias fraternas sobre la discapacidad intelectual, se han estudiado diferentes factores que parecen influir en la adaptación psicológica del hermano con discapacidad y, por tanto, en la relación fraternal. Entre estas variables se encuentra la gravedad de la discapacidad del hermano midiéndose mediante la existencia o no de problemas conductuales, ya que parece que es más complicada la adaptación en aquellos hermanos con una discapacidad intelectual profunda y con problemas externalizantes (Aparicio y Mínguez, 2015; Dyke, Mulroy y Leonard, 2009; Hastings, 2003). También se ha analizado la edad de los hermanos y la etapa en la que se encuentran, pues se ha conocido que estas experiencias pueden variar dependiendo de la persona y del momento del ciclo vital en el que esté, no siendo igual la experiencia de convivir con un hermano con discapacidad en la primera infancia que en la adultez. Además, las preocupaciones y retos a los que se tienen que enfrentar varían, destacando en la primera infancia sentimientos como el desconcierto, malestar y deseo de curación de los hermanos con discapacidad; en la adolescencia sentimientos de responsabilidad, culpa y vergüenza; y en la edad adulta sentimientos más positivos, aunque con una mayor preocupación por el futuro del hermano y por la carga genética que pudiera haber heredado (Lobato, 1992; Navarro, 2017; Scelles, 2006).

Otro factor que parece influir en la valoración de esta experiencia es la explicación que reciben los hermanos por parte de sus padres y profesionales sanitarios. Se ha detectado que el desconocimiento o ambigüedad ante una situación, crea inseguridad y estrés, y para lidiar con esto, los niños suelen fantasear pudiendo crear ideas erróneas y temores como que ellos han provocado la enfermedad de su hermano por su mal comportamiento, etc. Para conseguir una valoración más positiva y una mejor adaptación familiar es necesario que los padres les proporcionen respuestas a sus preguntas e información adaptada a la edad de cada niño (Harris, 2001). A su vez, el ambiente familiar también parece ser relevante, pues estudios previos muestran como las familias con un buen funcionamiento familiar y bajo índice de conflicto ayudan a tener menos problemas internalizantes y externalizantes en todos los miembros de la familia (Stoneman, 2006). 
De hecho, en entrevistas realizadas a hermanos adultos de personas con discapacidad intelectual, éstos señalan como tras su experiencia vivida (negativa o positiva) podría haber sido más satisfactoria si los profesionales y familiares hubieran atendido a necesidades como: estar informados sobre la enfermedad o trastorno de su hermano, su evolución y tratamientos y tener un espacio de expresión de sus emociones (Lizasoáin y Onieva, 2010).

\section{4. ¿ES DIFERENTE LA EXPERIENCIA DE TENER UN HERMANO CON SÍNDROME DE DOWN, AUTISMO O NEUROTÍPICO?}

En la literatura revisada existen diferentes posturas que responden esta cuestión, no estando claro si existen mayores o menores dificultades en el ajuste psicológico de los hermanos de niños con discapacidad. Por un lado, encontramos resultados que indican que los hermanos de niños con discapacidad tienen un peor ajuste psicológico que los hermanos de niños neurotípicos y resaltan entre ellos como peor adaptados psicológicamente a los hermanos de niños con autismo en comparación con hermanos neurotípicos o hermanos de niños con síndrome de Down (Aparicio y Mínguez, 2015; De Caroli y Sagone, 2013; Hastings, 2003; Orsmond y Seltzer, 2007; Pollard et al., 2013; Ross, y Cuskelly, 2006). Esto se evidencia en el estudio de Orsmond y Seltzer que concluye que los hermanos de niños autistas refieren pasar menos tiempo con sus hermanos en comparación con los hermanos de niños con síndrome de Down (Orsmond y Seltzer, 2007) y en una investigación realizada por De Caroli y Sagone (2013) donde se muestra que los hermanos de niños con autismo presentan un autoconcepto más negativo de sí mismos que los hermanos de niños con discapacidad intelectual. Estos resultados pueden ser debidos, como se viene mostrando a lo largo de los diferentes análisis, por las características propias del Trastorno Autista que dificultan las interacciones sociales.

Sin embargo, por otro lado, encontramos estudios cuyos resultados indican no encontrar diferencias significativas entre los hermanos de niños con discapacidad y los hermanos de niños neurotípicos siendo el ajuste emocional y conductual similar en ambos grupos (síndrome de Down, autismo y neurotípico) (Cuskelly y Gunn, 2006; Hastings, 2007; Hastings y Petalas, 2014; Moyson y Roeyers, 2012; Walton e Ingersoll, 2015). 


\subsection{OBJETO DEL ESTUdio}

A la vista de los antecedentes expuestos, el presente estudio se centra en los hermanos con discapacidad intelectual, pero sobre todo en dos grandes núcleos, como es el síndrome de Down (definido por la Clasificación Internacional de Enfermedades (CIE-10) como una alteración cromosómica del par 21 que se caracteriza por la presencia de discapacidad cognitiva y rasgos físicos peculiares) (Organización Mundial de la Salud, 2000) y el Trastorno del Espectro Autista con discapacidad (definido por el DSM-5 como alteraciones en el desarrollo que aparecen en la infancia y afectan a la comunicación verbal y no verbal, a la interacción social y flexibilidad de intereses) (American Psychiatric Association, 2013), ya que el 75\% de los casos de personas con autismo están vinculados con discapacidad intelectual.

Estos dos grupos han sido seleccionados por las diferencias que presentan entre sí, aun compartiendo ambos la discapacidad intelectual. Es interesante conocer si en el caso de encontrarse dificultades en las vivencias de los hermanos, éstas se deben a la discapacidad intelectual como variable categórica o a las características asociadas al trastorno o enfermedad del hermano. Hay que tener en cuenta que la discapacidad es un amplio espectro, y en la vivencia de ésta influye el grado de afectación, la existencia de un trastorno asociado y las características de éste. Por ello, si se tienen en cuenta todos estos factores, es coherente encontrar en la literatura mayores complicaciones en los hermanos de niños con autismo, por estar asociado a este trastorno un comportamiento menos adaptativo socialmente y dificultades comunicacionales que se presentan adicionales a la discapacidad.

La selección de los hermanos de los niños de estos dos grupos se justifica porque en ambos casos la afectación cognitiva aparece de forma muy temprana o incluso desde el nacimiento del niño, por lo que los hermanos de éstos convivirán con ellos durante su infancia involucrándose ya desde entonces en un proceso de adaptación familiar.

A la vista de los estudios mencionados anteriormente y las grandes discrepancias que existen, cabe preguntarse si tener un hermano con discapacidad intelectual provoca diferencias a la hora de resolver problemas, adaptarse y enfrentarse a la vida. Y en el caso de que existan, si estas diferencias se perciben como dificultades, fortalezas personales o ambas.

La presente investigación se centra en un análisis sobre las fortalezas, dificultades y ajuste psicológico que tienen los hermanos de niños con discapacidad. Para ello se han incluido variables tales como las siguientes: 
- El nivel socioeconómico. Cuanto mayor nivel socioeconómico presenta la familia, el nivel de ajuste y la percepción de bienestar es mayor (Aparicio y Mínguez, 2015; Dyke, Mulroy y Leonard, 2009).

- El sexo. Varían los resultados en función del estudio. Por un lado, las mujeres presentan peor nivel de ajuste psicológico por adquirir el rol de cuidadora y no solo el de hermana. Además, parece influir la etapa evolutiva, pues, aunque éstas se adapten mejor en la niñez, esto varía a largo plazo, ya que se observan más problemas psicológicos en ellas (Hallberg, 2013; Harris, 2001; Hastings, 2003). Por otro lado, estudios muestran que las mujeres presentan mejores niveles de ajuste psicológico y mayores habilidades de afrontamiento (Aparicio y Mínguez, 2015).

- El grado de problemas de conducta que presenta el hermano con discapacidad. Los estudios muestran que cuanto mayores son los problemas conductuales, peor es el ajuste psicológico del hermano (Aparicio y Mínguez, 2015; Cagigal y Prieto-Ursúa, 2006; Dyke et al., 2009; Hastings, 2003; Ross y Cuskelly, 2006).

- El orden que ocupa el hermano sin discapacidad en la jerarquía fraterna. Se han observado diferencias entre la posición que ocupa cada hermano siendo los hermanos menores de niños con trastorno los que tienden a experimentar un mayor desajuste. Además, cuanto mayor sea el número de hermanos, mejor será la adaptación psicológica de los hermanos neurotípicos, pues existen más hermanos con los que compartir esta experiencia de cuidado y con quienes expresarse emocionalmente (Aparicio, y Mínguez; Dyke, Mulroy y Leonard, 2009; Harris, 2001; Hastings, 2003).

- La percepción de los hermanos de la calidad de relación fraternal. Esta es una variable muy subjetiva a evaluar, pero se debe tener en cuenta, ya que puede presuponerse que los hermanos por el hecho de serlo tienen una relación adecuada entre sí difiriendo esto con la realidad. Dado que existen pocos estudios que contemplen la variable de la relación entre hermanos, esta investigación la tendrá en consideración, ya que presentan una mayor autoestima y ajuste psicológico cuando la calidad de la relación percibida entre los hermanos es mejor. Además, la relación percibida por los hermanos de niños con Trastorno Autista suele ser peor evaluada que los de aquellos con otros trastornos, pues en estos últimos, los hermanos muestran más interés por las relaciones y entre ellas las fraternales (Pollard et al., 2013). 
Se seleccionaron estas variables por ser las más significativas en los estudios revisados en relación con el ajuste psicológico. Otras variables como el clima familiar, la información recibida, los recursos y apoyo de cada familia también han demostrado influir en la adaptación de los hermanos, pero los estudios que miden estas variables son menores y por longitud y sobresaturación de esta población en las investigaciones, el análisis se ha centrado solo en las variables mencionadas anteriormente. Además, el grado de discapacidad del hermano afectado no ha sido evaluado por considerarse, tras la revisión de las investigaciones, menos relevante que los problemas de conducta de éste en el ajuste psicológico, y tratando, por tanto, en el estudio la discapacidad intelectual como variable categórica.

El objetivo general planteado en esta investigación es estudiar las posibles diferencias que pueden existir entre los hermanos de niños con síndrome de Down, autismo y neurotípicos respecto al ajuste psicológico de éstos ante la vida medido a través del análisis de sus dificultades y fortalezas sociales.

Los objetivos específicos del estudio son:

- Comprobar si existe relación entre los problemas de conducta del hermano con discapacidad y el ajuste psicológico fraterno.

- Determinar si existe relación entre el nivel socioeconómico de la familia y el ajuste psicológico de los hermanos sin discapacidad.

- Estudiar la relación entre la percepción de los hermanos de la calidad de relación fraternal y el ajuste psicológico de éstos.

- Determinar si existen diferencias entre los hombres y las mujeres en cuanto al ajuste psicológico.

- Comprobar si existen diferencias en el ajuste psicológico entre los hermanos que ocupan distinta posición en el orden de nacimiento.

- Estudiar si existen diferencias en los problemas de conducta entre los distintos grupos (niños con síndrome de Down, con autismo o neurotípicos).

Las hipótesis del estudio son:

- Existen diferencias significativas en las fortalezas y dificultades entre los hermanos de niños con discapacidad intelectual y los hermanos de personas neurotípicas. Se espera a su vez encontrar diferencias entre los hermanos de personas con estos diagnósticos, siendo los hermanos de niños con autismo los que presenten mayores dificultades de ajuste psicológico.

- Los problemas de conducta del hermano con discapacidad se relacionan con el ajuste psicológico fraterno, siendo mejor este ajuste cuanto menores son los problemas conductuales del hermano. 
- Hay relación entre el nivel socioeconómico de la familia y el ajuste psicológico de los hermanos sin discapacidad. Se espera encontrar una relación positiva, a mayor nivel socioeconómico, mayor ajuste psicológico de los hermanos, pues los recursos económicos y educativos son más elevados.

- Existe relación entre la percepción de los hermanos de la calidad de la relación fraternal y el ajuste psicológico de éstos. Cuanto mejor es la relación entre hermanos mejor será el ajuste.

- Hay diferencias entre los hombres y las mujeres en cuanto al ajuste psicológico. A pesar de las discrepancias entre los distintos estudios, se esperan encontrar diferencias en la variable sexo, siendo las mujeres las que presenten un mayor ajuste psicológico.

- Existen diferencias en el ajuste psicológico del hermano respecto a la posición en el orden de nacimiento, siendo los hermanos más mayores los que obtengan un ajuste psicológico mayor.

- Hay diferencias en los problemas de conducta entre los distintos tipos de trastorno o enfermedad (síndrome de Down, autismo y neurotípico). Se espera que los niños con autismo presenten mayores problemas conductuales, seguidos de los niños con síndrome de Down y de los que no presentan discapacidad intelectual.

\section{MÉTODO}

\subsection{Participantes}

Para la realización de esta investigación se ha accedido a una muestra de hermanos de niños neurotípicos y a hermanos de niños con síndrome de Down y con autismo con discapacidad intelectual todos ellos desde los 10 hasta los 20 años (ver Tabla 1). Para ello se ha contado con la participación de diversos casos de conocidos y allegados (muestreo incidental) que cumplen este requisito, colegios de educación especial (Colegio Público de Educación Especial Alfonso X el Sabio y Aula TGD del Instituto Público Julio Caro Baroja), centros y asociaciones de discapacidad intelectual, autismo o síndrome de Down de la Comunidad de Madrid (Asociación Autismo Araya, Grupo AMÁS, Asociación Argadini, APANSA, ADEMO, Plena Inclusión y Asociación ASPADO). Se ha pretendido aplicar los cuestionarios en diferentes áreas geográficas de la Comunidad de Madrid para así tener una muestra 
más amplia de la realidad y atender a la posible influencia de la variable socioeconómica.

Para la aplicación de estos cuestionarios se ha dispuesto de una muestra de niños siendo el $55 \%$ chicas $(\mathrm{N}=22)$ y $45 \%$ chicos $(\mathrm{N}=18)$, de los cuales el $60 \%$ formaban parte del grupo experimental de hermanos de niños con discapacidad intelectual, y a su vez estos se dividían en hermanos de niños con síndrome de Down (21\% chicos y $79 \%$ chicas) y hermanos de niños con autismo (siendo el 30\% chicas y el 70\% chicos). Por otro lado, el grupo control componía el $40 \%$ de la muestra, de los cuales el 50\% eran chicas y el $50 \%$ chicos. La edad de éstos era muy homogénea, pues la media de todos ellos es muy similar (ver Tabla 1).

Respecto al nivel socioeconómico, 42,5\% de los padres tienen unos ingresos entre 27.000-36.000 euros anuales frente a un 32,5\% de las madres. Las madres participantes en el estudio tienen menos ingresos que los padres, pero estos están distribuidos de manera más equilibrada entre las distintas categorías laborales, hay más homogeneidad que en los hombres. A su vez, hay un mayor porcentaje de mujeres que no trabajan $(32,50 \%)$ en comparación con los hombres (15\%).

TABLA 1. MEDIA Y DESVIACIÓN TÍPICA DE LA EDAD DE CADA GRUPO (HERMANOS DE NIÑOS CON SÍNDROME DE DOWN, HERMANOS DE NIÑOS CON AUTISMO, HERMANOS DE NIÑOS SIN PATOLOGÍA DE DISCAPACIDAD)

\begin{tabular}{|l|c|c|}
\hline & Media & D.T. \\
\hline Edad hermanos síndrome de Down & 16,79 & 1,477 \\
\hline Edad hermanos autismo & 15,70 & 4,165 \\
\hline Edad hermanos neurotípicos & 15,13 & 1,996 \\
\hline Total edad de los tres grupos & 15,85 & 2,607 \\
\hline
\end{tabular}

Nota. D.T.: desviación típica.

\subsection{INSTRUMENTOS}

En este estudio se han utilizado varios instrumentos, algunos de ellos completados por los padres de los niños con discapacidad y otros por los propios hermanos. El principal instrumento que se ha aplicado a los hermanos de niños con discapacidad intelectual es el SDQ (Strengths and Difficulties Questionnaire; Gómez-Beneyto et al, 2013) que consta de 25 ítems y se 
divide en cinco subescalas: síntomas emocionales, problemas de conducta, hiperactividad, problemas con compañeros y comportamientos prosociales. Este cuestionario evalúa estos aspectos atendiendo a su aparición en los últimos seis meses. Se evalúan en una escala de respuesta Tipo Likert siendo 0 «no es verdad», 1 «es verdad a medias» y 2 «verdaderamente si», a excepción de los ítems 7 "por lo general soy obediente», 21 «pienso las cosas antes de hacerlas», 25 "termino las tareas que estoy haciendo», 11 «tengo un buen/a amigo/a o más de uno/a» y 14 "por lo general caigo bien a otra gente de mi edad» que son inversos.

Se ha considerado pertinente la aplicación del cuestionario SDQ de autoinforme por tener evidencias de validez y fiabilidad en población española adolescente. En general, sus evidencias de fiabilidad mostraron niveles adecuados $(0,75)$, aunque en la subescala de problemas de conducta esta consistencia es más discreta, presentando valores inferiores a 0,75 en Alfa de Cronbach. En cuanto a la validez del cuestionario, este obtiene unos valores de sensibilidad y especificidad elevados: 0,96 y 0,95 respectivamente. La validez del constructo no muestra variabilidad del cuestionario ante distintas poblaciones ( $\mathrm{KMO}=0,78$ y la esfericidad $p<0,001)$. Además, este instrumento tiene la ventaja de ser breve y de resultar fácil a la hora de completarlo (Ortuño-Sierra et al., 2015).

Como ampliación del cuestionario SDQ se les ha presentado un ítem adicional: "¿Cómo defines la relación con tu hermano/a?», que mide la percepción de la calidad de la relación fraternal que tiene el hermano del niño con discapacidad en escala Tipo Likert (siendo 1 «muy mala» y 5 «muy buena») para así conocer la influencia que tiene en la adaptación psicológica de estos.

La mayoría de los estudios sobre discapacidad intelectual obtienen los problemas de conducta asociados como una variable muy significativa a evaluar en la relación de los hermanos, por lo que se ha considerado relevante la aplicación del cuestionario ICAP (Inventario para la Planificación de Servicios y Programación individual; Montero, 1993) sobre problemas externalizantes. Este cuestionario ha de ser completado por alguna persona que haya tenido contacto con el niño al menos durante tres meses, en este caso, por los padres del niño.

La escala de problemas de conducta del ICAP se subdivide en dos grandes grupos: conductas disociales cuando suponen una amenaza para sí mismo y/o para los demás y conductas no disociales cuando el problema conductual se deriva de la pasividad del sujeto restringiéndose o no participando en actividades. A su vez, estas conductas se clasifican en cuatro subescalas: problemas de conducta internos, externos, sociales y generales. Y dentro de 
estas categorías pueden ser clasificadas en siete tipos de problemas comportamentales medidos en frecuencia y gravedad: comportamientos autolesivos o daño a sí mismo, heteroagresividad o daño a otros, destrucción de objetos, conducta disruptiva, hábitos atípicos y repetitivos (estereotipias), conducta social ofensiva, retraimiento o falta de atención y conductas no colaboradoras.

La puntuación de este instrumento puede moverse en un rango desde -70 («muy grave») a 10 («normal») en las distintas subescalas, pudiéndose obtener así puntuaciones más negativas cuanto mayor es la gravedad de los problemas de conducta del sujeto.

En cuanto a la fiabilidad del ICAP, la investigación muestra una adecuada consistencia interna con valores entre 0,76 y 0,94 , fiabilidad test-retest $(0,93)$ y fiabilidad interjueces $(0,75)$. La validez del cuestionario ha mostrado ser satisfactoria discriminando adecuadamente entre los niños que presentan problemas de conducta y los que no con una puntuación de 0,81. Se ha determinado mediante un análisis discriminante que el 92\% de los sujetos estaban bien clasificados, diferenciándose así los niños con problemas de conducta de los niños con conductas adaptativas. Por lo tanto, la escala de problemas de conducta de ICAP ha evidenciado ser adecuada para evaluar los problemas de conducta por un observador del entorno del sujeto como son los padres (Montero, 1993).

\subsection{Procedimiento}

Se ha accedido a la muestra a través del contacto electrónico, telefónico y presencial con las distintas asociaciones, a las cuales se les hizo llegar los cuestionarios de forma electrónica (vía Internet) y en papel guardando el anonimato de éstos.

Las instrucciones para la aplicación del cuestionario fueron indicadas en el mismo enlace o papel precisando la necesidad de que el cuestionario que evalúa los problemas de conducta (ICAP) fuese rellenado por los padres atendiendo a las conductas de su hijo con discapacidad intelectual, y que el cuestionario de dificultades y fortalezas (SDQ) fuese completado por los hermanos de los niños con discapacidad. Se ha considerado importante que la aplicación del cuestionario haya sido respondida por los hermanos de niños con discapacidad y no por los padres para evitar posibles diferencias en las respuestas, ya que en ocasiones las percepciones de éstos sobre sus vivencias emocionales y psicológicas difieren entre sí (Moyson y Roeyers, 2012). 


\subsection{ANÁLISIS DE DATOS}

Para realizar el análisis estadístico de los datos se ha utilizado la versión 22 del software IBM $^{\circledR}$ SPSS $^{\circledR}$ (Statistical Package for the Social Sciences) (IBM Corp. Released, 2013).

La investigación planteada es un estudio ex post facto de tipo retrospectivo complejo, pues se tendrán en cuenta varias variables independientes para la explicación de los cambios en el ajuste psicológico (variables dependientes). Para la consecución de los objetivos mencionados, se realizarán análisis de diferencias entre grupos mediante análisis descriptivos (media, desviación típica para las variables cuantitativas y frecuencias y porcentajes para las variables cualitativas) y análisis inferenciales con medidas no paramétricas (prueba de Kurskal-Wallis y prueba de U de Mann-Whitney para los análisis de diferencias de grupos y Spearman para correlaciones) tras no cumplir el supuesto de Normalidad. También se realizó la prueba del tamaño del efecto $r$ de Rosenthal $(r=z / \sqrt{ } N)$ (1991).

\section{RESULTADOS}

\subsection{ANÁlisis DESCRIPTIVO DE SDQ (AJUSTE PSICOLÓGICO)}

En la Tabla 2, se muestran las medias en las dimensiones que componen el ajuste psicológico, donde se presentan mayores puntuaciones de los sujetos en la subescala de conductas prosociales (subescala que mide las fortalezas del ajuste psicológico) que en el resto de subescalas. Por lo tanto, a rasgos generales, los hermanos tienen un ajuste psicológico en el que predominan más sus fortalezas (conductas prosociales) que sus dificultades (problemas emocionales, de conducta, hiperactividad y problemas con los compañeros). 
TABLA 2. ANÁLISIS DESCRIPTIVOS DE LAS SUBESCALAS DEL CUESTIONARIO SDQ

\begin{tabular}{|l|c|c|c|c|}
\hline & Mínimo & Máximo & Media & D.T. \\
\hline Problemas emocionales & 0 & 9 & 2,98 & 2,496 \\
\hline Problemas de conducta & 0 & 5 & 1,92 & 1,207 \\
\hline Hiperactividad & 0 & 9 & 3,17 & 2,062 \\
\hline Problemas con los compañeros & 0 & 9 & 1,70 & 1,772 \\
\hline Conductas prosociales & 4 & 10 & 8,92 & 1,248 \\
\hline Total dificultades & 2 & 23 & 9,78 & 5,395 \\
\hline
\end{tabular}

Nota. D.T.: desviación típica; $\mathrm{N}=40$.

\subsection{Objetivo 1. Diferencias Entre tipo de trastorno o ENFERMEdAd y AJUSTE PSICOLÓGICO}

El objetivo del estudio era estudiar las posibles diferencias que pueden existir entre los hermanos de niños con síndrome de Down, autismo y neurotípicos respecto al ajuste psicológico. Para ello se ha llevado a cabo la prueba de Kruskal Wallis dado que no se cumplió el supuesto de Normalidad (test Kolmogorov-Smirnov $p<0,05$ ).

Según los resultados obtenidos no se presentan diferencias entre las subescalas del ajuste psicológico: dificultades emocionales $\left(\mathrm{X}^{2}(2)=4,523 ; p=\right.$ $0,104)$, problemas de conducta $\left(\mathrm{X}^{2}(2)=0,705 ; p=0,703\right)$, hiperactividad $\left(\mathrm{X}^{2}(2)=3,032 ; p=0,220\right)$, problemas con los compañeros $\left(\mathrm{X}^{2}(2)=0,914 ; p\right.$ $=0,633)$, conductas prosociales $\left(\mathrm{X}^{2}(2)=1,073 ; p=0,585\right)$ y las dificultades totales $\left(\mathrm{X}^{2}(2)=3,202 ; p=0,202\right)$ y el tipo de trastorno o enfermedad del hermano.

\subsection{Objetivo 2. Relación de PRoblemas de conducta DEL hermano con DISCAPACIDAD Y EL AJUSTE PSICOLÓGICO FRATERNO}

Se realizaron análisis de correlación de Spearman por tratarse de medidas no paramétricas. Tras su análisis no se encontró relación entre los problemas de conducta de las personas con discapacidad (problemas internalizantes, problemas sociales, problemas externalizantes, problemas de conducta generales) y el ajuste psicológico de sus hermanos (problemas 
emocionales, problemas de conducta, hiperactividad, conductas prosociales, problemas con los compañeros y dificultades totales) (ver Tabla 3).

TABLA 3. CORRELACIONES DE SPEARMAN ENTRE PROBLEMAS DE CONDUCTA (ICAP) Y AJUSTE PSICOLÓGICO (SDQ)

\begin{tabular}{|l|c|c|c|c|c|c|}
\hline & P. E. & P. C. & Hiperactividad & C. P. & P. C. & $\begin{array}{c}\text { Dificultades } \\
\text { totales }\end{array}$ \\
\hline $\begin{array}{l}\text { Problemas } \\
\text { internalizantes }\end{array}$ & $-0,32$ & 0,56 & 0,114 & 0,114 & 0,134 & 0,063 \\
\hline $\begin{array}{l}\text { Problemas } \\
\text { sociales }\end{array}$ & $-0,131$ & $-0,174$ & $-0,097$ & 0,093 & 0,127 & $-0,140$ \\
\hline $\begin{array}{l}\text { Problemas } \\
\text { externalizantes }\end{array}$ & $-0,095$ & 0,134 & 0,70 & 0,192 & 0,163 & 0,040 \\
\hline $\begin{array}{l}\text { Problemas } \\
\text { generales de } \\
\text { conducta }\end{array}$ & $-0,108$ & $-0,003$ & $-0,061$ & 0,060 & 0,192 & $-0,069$ \\
\hline
\end{tabular}

Nota. P. E.: Problemas Emocionales; P. C.: Problemas de Conducta; C. P.: Conducta Prosocial; P. C.: Problemas con Compañeros; $p$ : nivel crítico de significación $(<0,05) ; N=40$.

\subsection{ObJetivo 3. Relación ENTRE EL NIVEL SOCIOECONÓMICO DE LA FAMILIA Y} EL AJUSTE PSICOLÓGICO DE LOS HERMANOS SIN DISCAPACIDAD

Se ha encontrado una relación estadísticamente significativa entre el nivel socioeconómico y la subescala conductas prosociales $(\mathrm{r}=0,345 ; p=$ $0,029)$, siendo la relación positiva y baja, lo cual indica que cuanto más nivel socioeconómico, más fortalezas (conductas prosociales). Aquellos sujetos que viven en familias con un poder adquisitivo mayor presentan más comportamientos de acercamiento e interacción social positivos con beneficios para el otro.

No se hallaron correlaciones significativas con las otras subescalas que componen la escala ajuste psicológico: dificultades emocionales $(r=-0,169$; $p=0,297)$, problemas de conducta $(\mathrm{r}=-0,084 ; p=0,606)$, hiperactividad ( $\mathrm{r}$ $=0,054 ; p=0,743)$, problemas con los compañeros $(\mathrm{r}=0,033 ; p=0,838) \mathrm{y}$ dificultades totales $(r=-0,097 ; p=0,550)$. 
3.5. OBJETIVo 4. RELACión ENTRE LA PERCEPCiÓN DE LOS HERMANOS DE LA CALIDAD DE LA RELACIÓN FRATERNAL Y EL AJUSTE PSICOLÓGICO DE ÉSTOS

Tras el análisis de la correlación de Spearman se obtuvo la confirmación de que no existe relación entre la percepción del hermano sin discapacidad sobre su relación fraterna y el ajuste psicológico de éste: dificultades emocionales $(\mathrm{r}=0,176 ; p=0,278)$, problemas de conducta $(\mathrm{r}=-0,026 ; p=0,874)$, hiperactividad $(r=-0,068 ; p=0,679)$, problemas con los compañeros $(r=$ $-0,027 ; p=0,867)$, conducta prosocial $(\mathrm{r}=-0,084 ; p=0,607)$ y total dificultades $(r=0,063 ; p=0,698)$. Es decir, en este estudio se observa que no influye el tipo de percepción de la relación con su hermano para tener más o menos fortalezas o dificultades psicológicas.

\subsection{Objetivo 5. Diferencias ENTRE los hombres y las MUJERES EN CUANTO AL AJUSTE PSICOLÓGICO}

Para el análisis de diferencias de grupos por sexo en el ajuste psicológico se utilizó la prueba estadística U de Mann-Whitney. Tras los resultados obtenidos se evidencia que no existen diferencias significativas en el ajuste psicológico: dificultades emocionales $(\mathrm{U}=166,5$; $\mathrm{z}=-0,866$; $p=0,387$ ), problemas de conducta $(\mathrm{U}=153 ; \mathrm{z}=-1,269 ; p=0,204)$, hiperactividad $(\mathrm{U}=173$; $\mathrm{z}=-0,691 ; p=0,489)$, problemas con los compañeros $(\mathrm{U}=194 ; \mathrm{z}=-0,112$; $p=0,911)$, conductas prosociales $(\mathrm{U}=183 ; \mathrm{z}=-0,430 ; p=0,667) \mathrm{y}$ las dificultades totales $(\mathrm{U}=188,5 ; \mathrm{z}=-0,259 ; p=0,795)$ entre hombres y mujeres.

3.7. ObJetivo 6. Diferencias ENTRE La POSICIÓN QUE OCUPA EL HERMANO DE LA PERSONA CON DISCAPACIDAD Y EL AJUSTE PSICOLÓGICO

Se utilizó el test de Kruskal-Wallis. Se encontró que no hay diferencias estadísticamente significativas entre el ajuste psicológico: dificultades emocionales $\left(\mathrm{X}^{2}(2)=0,117 ; p=0,943\right)$, problemas de conducta $\left(\mathrm{X}^{2}(2)=0,895\right.$; $p=0,693)$, hiperactividad $\left(\mathrm{X}^{2}(2)=0,683 ; p=0,711\right)$, problemas con los compañeros $\left(\mathrm{X}^{2}(2)=1,584 ; p=0,453\right)$, conductas prosociales $\left(\mathrm{X}^{2}(2)=0,969 ; p=\right.$ $0,616)$ y las dificultades totales $\left(\mathrm{X}^{2}(2)=0,305 ; p=0,859\right)$ y la posición que ocupa el hermano sin discapacidad en la jerarquía fraterna. 
3.8. Objetivo 7. Diferencias en los problemas de conducta entre los DisTintos GRUPOS (SínDROME DE Down, AUTISMO y NEURoTíPICOS)

Al igual que en el análisis anterior, se utilizó el test de Kruskal-Wallis. Según esta prueba no existen diferencias significativas entre la subescala de los problemas externalizantes y los distintos grupos (niños con síndrome de Down, autismo y sin discapacidad $)\left(\mathrm{X}^{2}(2)=2,046 ; p=0,359\right)$, pero si se observan diferencias estadísticamente significativas entre el resto de subescalas: problemas internalizantes $\left(\mathrm{X}^{2}(2)=6,488 ; p=0,039\right)$, problemas sociales $\left(\mathrm{X}^{2}(2)=6,884 ; p=0,032\right)$, problemas generales $\left(\mathrm{X}^{2}(2)=9,747 ; p=\right.$ $0,008)$ y los distintos grupos.

\section{FIGURA 1. COMPARACIÓN DE MEDIANAS EN LOS DISTINTOS GRU- POS (SÍNDROME DE DOWN, AUTISMO Y NEUROTÍPICO)}

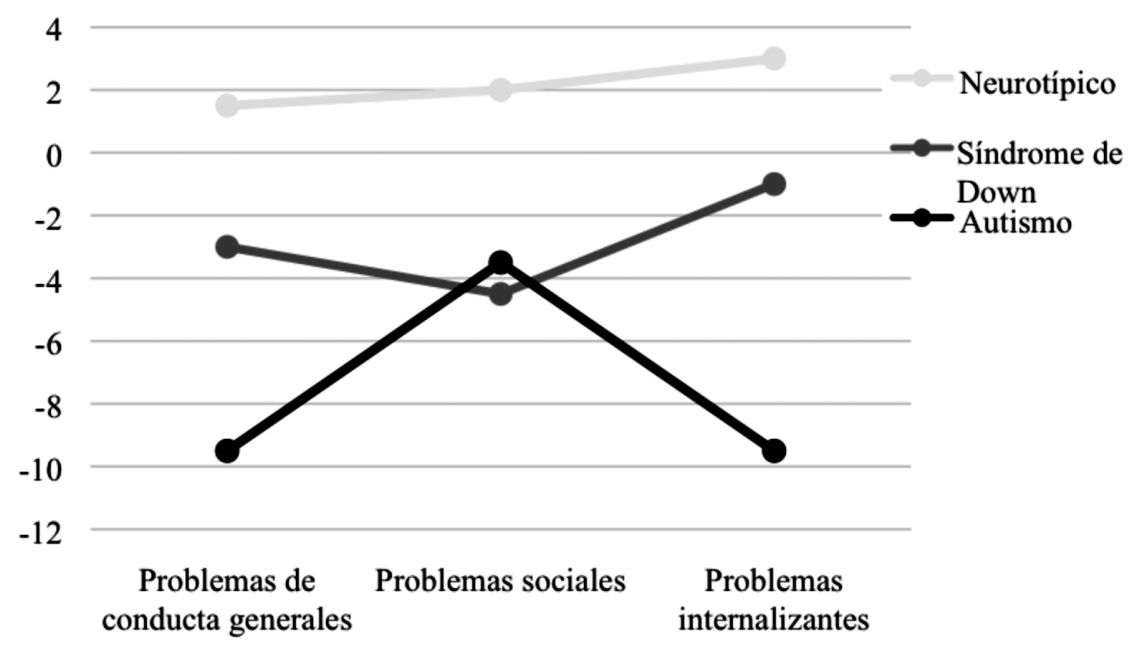

Estas diferencias se siguieron realizando test U de Mann-Whitney con comparaciones dos a dos para observar entre qué grupos se encontraban estas discrepancias. No se utilizó la corrección de Bonferroni, ya que la muestra del estudio era muy pequeña. En el caso de utilizarse esta corrección se encontrarían diferencias significativas únicamente entre los grupos de autistas y niños sin discapacidad en la subescala problemas generales $(0,001<0,017)$ y los grupos de niños con síndrome de Down y niños neurotípicos en la subescala de problemas sociales $(0,014<0,017)$. 
Se han encontrado diferencias estadísticamente significativas en la subescala del cuestionario ICAP de problemas generales entre los niños sin discapacidad y los niños con Trastorno Autista con un tamaño del efecto alto $(\mathrm{U}=19 ; \mathrm{z}=-3,234 ; p=0,001 ; \mathrm{r}=-0,634)$. Siendo la Mediana del grupo de niños neurotípicos de 1,5 y del grupo de autistas de -9,5, es decir, presentan mayores problemas de conducta generales los niños autistas por tener una puntuación menor que el grupo de niños sin discapacidad (ver Tabla 4). No se hallaron diferencias en esta subescala entre los grupos de neurotípicos con el grupo de síndrome de Down y entre el grupo de autistas y éste último.

También se encontraron diferencias entre los niños neurotípicos y aquellos con síndrome de Down en la subescala del cuestionario ICAP de problemas sociales $(U=56,5 ; z=-2,351 ; p=0,014 ; r=-0,429)$ con un tamaño del efecto moderado y con una Mediana de 2 en el grupo de niños neurotípicos y de $-4,5$ en el grupo de niños con síndrome de Down (ver Tabla 4). El grupo de niños sin discapacidad presenta menos problemas sociales que los niños con síndrome de Down al tener mayor puntuación en su Mediana (Mediana $=2>-4,5)$. No se encontraron diferencias en el resto de grupos entre sí.

Según los resultados obtenidos, existen diferencias en la subescala del cuestionario ICAP de problemas internalizantes (problemas de conducta hacia el propio sujeto: retraimientos, conductas estereotipadas y autoagresiones) entre los grupos niños con autismo y los que no presentan discapacidad intelectual con un tamaño del efecto moderado $(U=43,5 ; z=-2,026$; $p=0,043 ; \mathrm{r}=-0,397)$ y entre los que padecen síndrome de Down y los niños neurotípicos con un tamaño del efecto moderado $(\mathrm{U}=61 ; \mathrm{z}=-2,302 ; p=$ 0,021; $r=-0,42)$. La Mediana más alta se encuentra en el grupo de niños sin discapacidad (Mediana $=3$ ) seguida del grupo de niños con síndrome de Down (Mediana = -1) y del grupo de autismo (Mediana = -9,5), por lo que los que padecen autismo presentan más problemas internalizantes que aquellos sin discapacidad, y a su vez, los niños con síndrome de Down muestran mayores problemas internalizantes que los niños neurotípicos (ver Tabla 4). 
TABLA 4. MEDIANAS DE LOS DISTINTOS GRUPOS (SÍNDROME DE DOWN, AUTISMO, NEUROTÍPICOS) EN LAS SUBESCALAS ICAP SIGNIFICATIVAS (PROBLEMAS DE CONDUCTA GENERALES, PROBLEMAS SOCIALES, PROBLEMAS INTERNALIZANTES)

\begin{tabular}{|l|l|c|}
\hline \multirow{3}{*}{ Problemas de conducta generales } & Grupos & Mediana \\
\hline \multirow{2}{*}{ Problemas sociales } & Autismo & $-9,5$ \\
\cline { 2 - 3 } & Neurotípico & 1,5 \\
\hline \multirow{3}{*}{ Problemas internalizantes } & Síndrome de Down & $-4,5$ \\
\cline { 2 - 3 } & Neurotípico & 2 \\
\hline & Síndrome de Down & -1 \\
\cline { 2 - 3 } & Autismo & $-9,5$ \\
\cline { 2 - 3 } & Neurotípico & 3 \\
\hline
\end{tabular}

Nota. Cuanto menor es la puntuación de la mediana, mayor es el nivel de gravedad de los problemas de conducta.

\section{DISCUSIÓN}

El objetivo principal de este estudio es analizar si existen diferencias en las fortalezas y dificultades de los hermanos de niños con síndrome de Down, autismo y sin discapacidad. La hipótesis propuesta supone que los hermanos de niños con autismo tendrán un peor ajuste psicológico que los de síndrome de Down y neurotípicos por las dificultades sociales que conlleva este trastorno en sí y, por tanto, con el hermano. Existen numerosos estudios que apoyan esta hipótesis y que confirman que los hermanos de niños con discapacidad tienen un peor ajuste psicológico que los hermanos de niños sin discapacidad (Aparicio y Mínguez, 2015; De Caroli y Sagone, 2013; Hastings, 2003; Orsmond y Seltzer, 2007; Pollard et al., 2013; Ross, y Cuskelly, 2006). Sin embargo, en la realización de este estudio se aceptó la hipótesis nula, confirmando que no existen diferencias significativas entre estos grupos de hermanos en cuanto a sus fortalezas y dificultades, coincidiendo con estudios previos que defienden la semejanza entre estos tres grupos en su ajuste psicológico (Cuskelly y Gunn, 2006; Hastings, 2007; Hastings y Petalas, 2014; Moyson y Roeyers, 2012; Walton e Ingersoll, 2015). Existen, por tanto, resultados muy dispares que pueden estar influidos por los criterios de los autores, el análisis de diferentes variables, el control de variables extrañas, el tamaño de la muestra, etc. que pueden justificar estas discrepancias. 
Como segundo objetivo se ha comprobado si se relacionan los problemas de conducta del hermano con discapacidad con el ajuste psicológico fraterno, no encontrándose relación entre estas variables. Son múltiples los estudios que afirman que el grado de problemas de conducta del hermano con discapacidad intelectual afecta al ajuste psicológico del otro (Aparicio y Mínguez, 2015; Cagigal y Prieto-Ursúa, 2006; Dyke et al., 2009; Hastings, 2003; Ross y Cuskelly, 2006) siendo peor el ajuste cuanto mayor es el grado de problemas de conducta. Los resultados obtenidos en esta investigación difieren de los esperados, y esto puede ser debido a una percepción paternal más positiva de los problemas conductuales de su hijo. El cuestionario ICAP pide que se detalle el tipo de problemática que se presenta y mide los problemas conductuales en gravedad y frecuencia. Es relevante destacar como una gran mayoría de las respuestas de los padres de niños con autismo clasifican las conductas de su hijo como problemáticas y con frecuencias elevadas, pero no las consideran de gran gravedad. Por tanto, el papel de la percepción individual es necesario tenerlo en cuenta, ya que en algunos casos los padres pueden estar acostumbrados a ciertas conductas y aunque consideran que son problemáticas, pueden percibirlas con menor gravedad de la que la percibiría un padre con hijos sin discapacidad. Esto, es completamente lógico, y es que cuando se tiene un hijo con algún tipo de trastorno o enfermedad, se aprende a integrar esas diferencias con el resto de niños como algo propio de éste y no se sienten como problemáticas sino como características, a no ser que sean muy invasivas.

El tercer objetivo es determinar si el nivel socioeconómico de la familia se relaciona con el ajuste psicológico de los hermanos sin discapacidad. Tras los resultados se acepta la hipótesis alterna, confirmando que sí existe relación entre estas dos variables. El nivel socioeconómico ha demostrado ser, en diferentes estudios, un elemento de influencia en el tipo de ajuste psicológico, pues por lo general cuantos más recursos económicos se tiene, mayor es la ayuda de la que la familia puede disponer, el cuidado del hijo con discapacidad suele estar más repartido y, por tanto, hay mayor adaptación psicológica. De esta manera, tal y como muestran los resultados obtenidos, la escala de fortalezas (conductas prosociales) está relacionada con un mayor nivel socioeconómico medido mediante el nivel de estudios y puesto de trabajo, lo que implica un mayor nivel cultural y bienestar familiar (Aparicio y Mínguez, 2015; Dyke, Mulroy y Leonard, 2009).

El siguiente objetivo consiste en estudiar la relación entre la percepción de los hermanos de la calidad de relación fraternal y el ajuste psicológico de éstos (Pollard et al., 2013). No se obtuvo relación estadísticamente significativa entre tener peor relación con los hermanos con discapacidad y tener 
un peor ajuste psicológico y viceversa. A pesar de que este resultado no era esperable en la investigación, éste puede ser explicado por la ausencia de respuestas de percepción negativa sobre la relación fraternal. Es decir, a excepción de un sujeto que percibió su relación como "muy mala”, el resto entró en el rango de "muy buena", "buena" o "regular", por lo que no hay variabilidad suficiente como para poder conocer si en realidad la relación de estos afecta al ajuste psicológico.

En el quinto objetivo no se confirma la existencia de diferencias entre los hombres y las mujeres en cuanto al ajuste psicológico. En relación con este objetivo, existen distintas investigaciones que señalan que sí existen diferencias entre ser hombre o mujer y la adaptación de éstos. Algunos resaltan que las mujeres tienen un mejor ajuste psicológico que los hombres, es decir que la escala de fortalezas (escala prosocial) debería tener puntuaciones mayores en mujeres que en hombres y las fortalezas puntuaciones menores (Aparicio y Mínguez, 2015). Otros, sin embargo, muestran como las mujeres tienen peor ajuste al adquirir más responsabilidades con respecto al cuidado de su hermano con discapacidad (Hallberg, 2013; Harris, 2001; Hastings, 2003). Por tanto, existen diferencias entre unos autores y otros. Los resultados de esta investigación podrían explicarse porque esta situación de responsabilidad y de cuidado por parte de la hermana puede haber cambiado de investigaciones anteriores a la propia, ya que actualmente el rol de hermana no implica necesariamente un cuidado exclusivo del sexo masculino como sucedía anteriormente. Por lo que no tiene por qué suponer una diferencia o cambio psicológico el hecho de ser hombre o mujer con la adaptación psicológica.

En el siguiente objetivo se quiso encontrar diferencias entre la posición que ocupa el hermano de la persona con discapacidad en cuanto al ajuste psicológico. Los resultados en este objetivo diferían de los esperados, no encontrándose diferencias significativas entre la posición jerárquica y las fortalezas y dificultades de éstos. Los estudios plantean que cuanto más mayor es el hermano, su ajuste será más adecuado, y que ocupar las posiciones más elevadas en la jerarquía fraterna otorga mayores fortalezas personales (Aparicio, y Mínguez; Dyke, Mulroy y Leonard, 2009; Harris, 2001; Hastings, 2003). Tras los resultados obtenidos se muestra como en este estudio esto no se confirma, siendo independientes entre sí la variable del ajuste psicológico y la posición fraterna. Esto puede ser debido a que tal y como se menciona en varias investigaciones, las dificultades y fortalezas pueden estar relacionadas con otras variables dinámicas, como el funcionamiento o interacción familiar y, por tanto, las diferencias entre ser hermano mayor o menor no existen, pues todos tienen un funcionamiento 
similar por pertenecer a la misma familia (Moyson y Roeyers, 2012; Stoneman, 2006).

El último objetivo que se analizó en esta investigación es estudiar si existen diferencias en los problemas de conducta entre los distintos grupos (niños con síndrome de Down, con autismo o neurotípicos). Tras el análisis correspondiente, al igual que en estudios revisados, se confirma esta hipótesis para algunas de las subescalas de los problemas de conducta, encontrándose diferencias en los problemas de conductas generales (subescala problemas generales) entre los niños autistas y niños sin discapacidad y siendo mayores los problemas en los niños con autismo (Aparicio y Mínguez, 2015; Dyke, Mulroy y Leonard, 2009; Hastings, 2007; Ross y Cuskelly, 2006). A su vez, existen diferencias en la subescala de problemas de conducta sociales entre los que padecen síndrome de Down y los que no padecen ningún tipo de discapacidad, siendo estos primeros los que presentan menores puntuaciones, es decir, presentan más problemas de conducta. Es llamativo encontrar diferencias en los problemas de conducta sociales entre estos dos grupos y no en el grupo de niños autistas, siendo éste el trastorno que más dificultades sociales conlleva. Esto puede ser, como ya se ha mencionado anteriormente, por la percepción paterna como criterio, ya que a los padres de hijos con síndrome de Down les supondrá más fácil fijarse en las dificultades sociales que puedan presentar sus hijos a diferencia de los padres de niños con autismo, que ya dan por hecho que en sí el trastorno conlleva unas dificultades sociales propias y puede no parecerles extraño.

Por último, en relación con este objetivo, encontramos diferencias significativas en la subescala de problemas de conducta internalizantes (retraimientos, conductas estereotipadas y autoagresiones) entre los niños con autismo y sin discapacidad y entre aquellos que padecen síndrome de Down y los que no presentan ningún trastorno, teniendo más problemas de conducta en los grupos con autismo o síndrome de Down por tener puntuaciones menores en sus Medianas. Esto puede ser debido a la propia discapacidad intelectual, pues las personas que padecen tanto síndrome de Down como autismo normalmente presentan más dificultades en la comunicación, expresión de sus necesidades y autorregulación, lo que en ciertos momentos los puede llevar a recurrir a las autoagresiones y estereotipias como manera de control ante los problemas de expresión e interacción con el otro (De Caroli y Sagone, 2013; Pollard et al., 2013).

Este estudio presenta varias limitaciones y tenerlas en cuenta podrían ayudar a entender algunos resultados obtenidos. En primer lugar, se disponía de una limitación muestral, lo que ha podido afectar a los resultados de la investigación, ya que se contaba con serias dificultades para acceder 
a la muestra de hermanos de niños con discapacidad. Estas poblaciones clínicas presentan sobresaturación para la participación en estudios psicológicos, médicos, estadísticos... por lo que acceder a ellos es una ardua tarea. También, se han tenido en cuenta otras limitaciones como la influencia de otras variables familiares no contempladas en el estudio, como pueden ser la adecuación del ambiente familiar, los recursos a los que las familias tienen acceso, los apoyos personales o institucionales, la educación recibida acerca del trastorno o enfermedad que padece su hermano, la relación familiar, etc. Estas variables pueden afectar al ajuste psicológico del hermano y, por tanto, sería de gran utilidad poder tenerlas en cuenta para futuros estudios con esta población. A su vez, sería interesante contemplar la posible limitación por el sesgo de deseabilidad social, ya que los participantes del estudio por el hecho de participar en una investigación pueden responder a los cuestionarios de manera socialmente adecuada o deseable, pudiendo alterar los resultados de las escalas de problemas de conducta del niño con discapacidad (ICAP) y disminuyendo los valores de estos o variando los niveles de dificultades y fortalezas en el cuestionario SDQ respectivamente.

Otra limitación que señalar es la puntuación de la fiabilidad del cuestionario SDQ para la subescala problemas de conducta, ya que presenta una consistencia interna por debajo de lo necesario ( $<0,75$ Alfa de Cronbach), lo que podría indicar una imprecisión en la medida de los problemas conductuales de los hermanos.

A pesar de las limitaciones en los resultados presentados, este estudio arroja una ficha más en este saco de diversidad de conclusiones, apoyando la similitud psicológica entre los grupos de hermanos con y sin discapacidad. Además, cabe destacar en este estudio la importancia que muestran las puntuaciones en problemas de conducta de los niños con síndrome de Down y autismo en comparación con los niños sin discapacidad, para incidir y aportar más ayuda profesional en esta área. Conocer estas dificultades encamina a los profesionales de la psicología en la dirección para averiguar qué necesidades y/o preocupaciones pueden presentar estas familias (cómo manejar los problemas conductuales, cómo explicarle esto al resto de familiares...). A su vez, tras las diferencias mostradas en distintos estudios, conviene atender a las necesidades de estos y utilizar recursos que han sido demostrados como beneficiosos para los hermanos de niños con discapacidad como pueden ser los talleres de hermanos donde tienen un espacio propio para reunirse con sus iguales y poder expresar sus emociones, necesidades y preocupaciones, sintiéndose comprendidos por compartir todos ellos la característica familiar común (Aparicio y Mínguez, 2015; 
Cagigal y Prieto-Ursúa, 2006; Hallberg, 2013; Iriarte e Ibarrola-García, 2016; Lizasoáin y Onieva, 2010; Núñez y Rodríguez, 2004; Rumeu, 2009).

Para futuras investigaciones sería de gran utilidad realizar más estudios para llegar a un consenso en los resultados sobre si es o no diferente la experiencia de tener un hermano con discapacidad intelectual en cuanto al ajuste psicológico. Además, sería interesante poder contar con muestras más amplias para poder realizar análisis más fiables y significativos y poder introducir algunas variables mencionadas anteriormente que pueden afectar a los resultados y, por tanto, al ajuste psicológico de estos hermanos. También, sería necesario realizar estudios longitudinales en los que se pueda valorar el estado psicológico de estos hermanos en distintas etapas de la vida y su evolución, para ver si sus fortalezas y dificultades aumentan, disminuyen o se estabilizan a lo largo de su desarrollo y así poder realizar apoyos psicológicos más acertados con esta población.

Como punto final es conveniente destacar como, a la vista de los resultados obtenidos en esta investigación donde no se aprecian diferencias significativas entre los grupos de niños con y sin discapacidad intelectual, pone de relieve la necesidad de revisar estudios anteriores que afirman lo contrario, pues de ser así, esta visión provoca un cambio de perspectiva, encaminado hacia las necesidades individuales de cada hermano y no a la suposición de dificultades en el ajuste psicológico de éstos.

\section{REFERENCIAS}

American Psychiatric Association (2013). Diagnostic and Statistical Manual of Mental Disorders ( $5^{a} \mathrm{ed}$.). Washington DC: American Psychiatric Association.

Aparicio, R. R., y Mínguez, R. T. (2015). El ajuste conductual, emocional y social en hermanos de niños con un trastorno del espectro autista. Una revisión teórica. Papeles del Psicólogo, 36(3), 189-197.

Bank, S., y Kahn, M. D. (1982). Intense sibling loyalties. En M. E. Lamb y B. SuttonSmith (Eds.) Sibling relationships: Their nature and significance across the lifespan, (pp. 251-266). Hillsdale, NJ: Lawrence Erlbaum Associates.

Cagigal, V. y Prieto-Ursúa, M. (2006). Problemas emocionales y de conducta en hermanos de menores con diagnóstico de trastorno mental. Clínica y Salud, 17(1), 51-68.

Cuskelly, M., y Gunn, P. (2006). Adjustment of children who have a sibling with Down syndrome: Perspectives of mothers, fathers and children. Journal of Intellectual Disability Research, 50(12), 917-925.

De Caroli, M. E., y Sagone, E. (2013). Siblings and disability: A study on social attitudes toward disabled brothers and sisters. Procedia-Social and Behavioral Sciences, 93, 1217-1223. 
Dyke, P., Mulroy, S., y Leonard, H. (2009). Siblings of children with disabilities: challenges and opportunities. Acta Pediatrica, 98(1), 23-24.

Gómez-Beneyto, M., Nolasco, A., Moncho, J., Pereyra-Zamora, P., Tamayo-Fonseca, N., Munarriz, M., Salazar, J., Tabarés-Seisdedos, R., y Girón, M. (2013). Psychometric behaviour of the strengths and difficulties questionnaire (SDQ) in the Spanish national health survey 2006. BMC psychiatry, 13(1), 95.

Hallberg, U. (2013). Situation and psychosocial well-being of older sisters to children with disabilities or chronic illnesses-the forgotten children? International Journal of Qualitative Studies on Health and Well-being, 8.

Harris, S. L. (2001). Los hermanos de niños con autismo. Su rol específico en las relaciones familiares. Madrid: Narcea.

Hastings, R. P. (2003). Brief Report: Behavioral Adjustment of Siblings of Children with Autism. Journal of Autism and Developmental Disorders, 33(1), 99-104.

- (2007). Longitudinal relationships between sibling behavioral adjustment and behavior problems of children with developmental disabilities. Journal of autism and developmental disorders, 37(8), 1485.

Hastings, R. P., y Petalas, M. A. (2014). Self-reported behaviour problems and sibling relationship quality by siblings of children with autism spectrum disorder. Child: care, health and development, 40(6), 833-839.

IBM Corp. Released (2013). IBM SPSS Stadistics for Windows. Version 22.0. Amonk, NY: IBM.

Iriarte, C., e Ibarrola-García, S. (2010). Bases para la intervención emocional con hermanos de niños con discapacidad intelectual. Electronic Journal of Research in Educational Psychology, 8(20), 373-410.

- (2016). Revisión de estudios sobre la vivencia emocional de la discapacidad intelectual por parte de los hermanos. Estudios Sobre Educación, 19, 53-75.

Latorre, G. (2014). SOS... Mi hermano es síndrome de Down. Madrid: Ediciones Pirámide.

Lizasoáin, O., y Onieva, C. E. (2010). Un estudio sobre la fratria ante la discapacidad intelectual. Intervención Psicosocial, 19(1), 89-99.

Lobato, D. (1992). Hermanos y hermanas de niños con necesidades especiales. Madrid: IMSERSO (Instituto de Migraciones y Servicios Sociales).

Montero, D. (1993). Evaluación de la conducta adaptativa en personas con discapacidad. Adaptación y validación del ICAP. Bilbao: ICE. Universidad de Deusto-Mensajero.

Moyson, T., y Roeyers, H. (2012). 'The overall quality of my life as a sibling is all right, but of course, it could always be better'. Quality of life of siblings of children with intellectual disability: the siblings' perspectives. Journal of Intellectual Disability Research, 56(1), 87-101.

Navarro, M. (2017). En el olvido: Hermanos y hermanas de personas con discapacidad intelectual (trabajo fin de grado). Universidad Pública de Navarra, España.

Núñez, B. (2003). La familia con un hijo con discapacidad: sus conflictos vinculares. Archivos Argentinos de Pediatría, 101(2), 133-142.

Núñez, B., y Rodríguez, L. (2004). Los hermanos de personas con discapacidad: una asignatura pendiente. Argentina: Asociación Amar. 
Núñez, B. A., Rodríguez, L., y Lanciano, S. (2005). El vínculo fraterno cuando uno de los hermanos tiene discapacidad. Siglo Cero, 36(216), 50-74.

Oñate, L., y Calvete, E. (2017). Una aproximación cualitativa a los factores de resiliencia en familiares de personas con discapacidad intelectual en España. Psychosocial Intervention, 26(2), 93-101.

Organización Mundial de la Salud (2000). CIE 10. Décima Revisión de la Clasificación Internacional de Las Enfermedades. Trastornos Mentales y del Comportamiento: Descripciones Clínicas y pautas para el Diagnóstico. Madrid: Meditor.

Organización Mundial de la Salud (2011). Banco Mundial. Informe mundial sobre la discapacidad. Ginebra: OMS.

Orsmond, G. I., y Seltzer, M. M. (2007). Siblings of individuals with autism or Down syndrome: Effects on adult lives. Journal of Intellectual Disability Research, 51(9), 682-696.

Ortuño-Sierra, J., Fonseca-Pedrero, E., Paino, M., Sastre i Riba, S., y Muñiz, J. (2015). Screening mental health problems during adolescence: Psychometric properties of the Spanish version of the Strenghts and Difficulties Questionnaire. Journal of Adolescence, 38, 49-56.

Plena Inclusión (2017). La discapacidad intelectual en cifras. Madrid: Confederación Plena Inclusión España. Recuperado de http://www.plenainclusion.org/discapacidad-intelectual/la-discapacidad-intelectual-en-cifras

Pollard, C. A., Barry, C. M., Freedman, B. H., y Kotchick, B. A. (2013). Relationship quality as a moderator of anxiety in siblings of children diagnosed with autism spectrum disorders or Down syndrome. Journal of Child and Family Studies, 22(5), 647-657.

Riggio, H. R. (2000). Measuring attitudes toward adult sibling relationships: The lifespan sibling relationship scale. Journal of Social and Personal Relationships, 17(6), 707-728.

Rosenthal, R. (1991). Meta-analytic procedures for social research (2nd ed.). Newbury Park, CA: Sage.

Ross, P., y Cuskelly, M. (2006). Adjustment, sibling problems and coping strategies of brothers and sisters of children with autistic spectrum disorder. Journal of Intellectual and Developmental Disability, 31(2), 77-86.

Rumeu, O. L. (2009). Discapacidad y familia: el papel de los hermanos. En El largo camino hacia una educación inclusiva: la educación especial y social del siglo XIX a nuestros días: XV Coloquio de Historia de la Educación, Pamplona-Iruñea, 29, 30 de junio y 1 de julio de 2009 (pp. 653-660). Universidad Pública de Navarra.

Scelles, R. (2006). La riqueza del vínculo fraterno. Revista Síndrome de Down, 23, 129-135.

Stoneman, Z. (2006). Hermanos de niños con discapacidad: Temas de investigación. Siglo cero, 37(219), 13-30.

Walton, K. M., y Ingersoll, B. R. (2015). Psychosocial adjustment and sibling relationships in siblings of children with autism spectrum disorder: risk and protective factors. Journal of autism and developmental disorders, 45(9), 2764-2778.

Watson, M. D. V. (2017). El estado actual de la investigación y la intervención sobre la vivencia emocional de los hermanos ante la discapacidad intelectual. Redes, $35,71-82$. 